\title{
ELLIPTICALS AND BARS: CENTRAL MASSES AND FRICTION
}

\author{
J. A. SELLWOOD \\ Department of Physics $\&$ Astronomy, Rutgers University \\ 136 Frelinghuysen Road, Piscataway, NJ 08854-8019, USA \\ E-mail: sellwood@physics.rutgers.edu
}

\begin{abstract}
I give a very brief review of aspects of internal dynamics that affect the global shape of a galaxy, focusing on triaxiality, bars and warps. There is general agreement that large central masses can destroy triaxial shapes, but recent simulations of this process seem to suffer from numerical difficulties. Central black holes alone are probably not massive enough to destroy global triaxiality, but when augmented by star and gas concentrations in barred galaxies, the global shape may be affected. Even though we do not understand the origin of bars in galaxies, they are very useful as probes of the dark matter density of the inner halo. Finally, I note that dynamical friction acts to reduce a misalignment between the spin axes of the disk and halo, producing a nice warp in the outer disk which has many of the properties of observed galactic warps.
\end{abstract}

\section{Introduction}

I was asked by the organizers to discuss the huge topic of what $N$-body simulations can tell us about the shapes of galaxies. As a comprehensive survey would be voluminous, I here restrict myself to a few internal evolutionary processes affecting galaxy shapes where there have been noteworthy recent developments. I also point out some areas where a lot more work is required. Other speakers describe aspects of galaxy formation, and the properties of collapsed halos.

\section{Triaxiality and central masses}

Soon after Schwarzschild (1979) showed that a large fraction of the stars must pursue box orbits to support a triaxial mass distribution in an elliptical galaxy, it was realized that a central mass, or just a density cusp, might destroy triaxiality. The reason, most clearly articulated by Gerhard \& Binney (1985), is that a box orbit carries a star arbitrarily close the center, where a central mass will deflect it through a large angle, scattering the star into an orbit of different shape. The loss of stars on the structure-supporting box orbits must change the global shape of the galaxy, at least in the inner regions. Only tube orbits, which avoid the center, remain once the potential becomes axisymmetric and the central mass concentration will no longer drive evolution. This general expectation has been confirmed in $N$-body simulations, beginning with the 

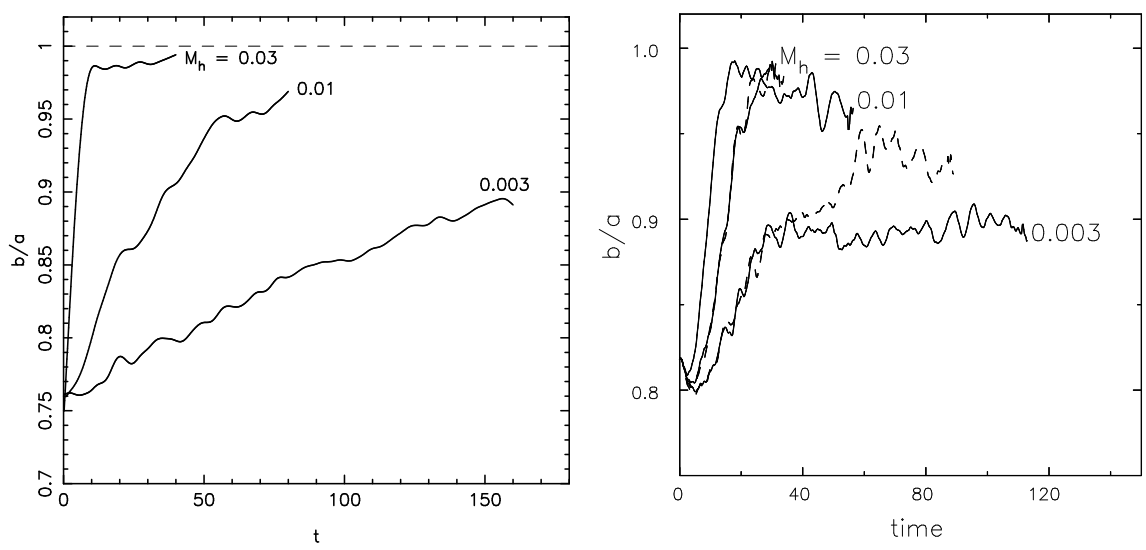

Figure 1: Comparison between my results (right) and those obtained by MQ98 (left). The plotted quantity is the ratio of the intermediate- to long-axes of the moment of inertia tensor of the $50 \%$ most bound particles as a function of time. The three solid curves show the results from models into which three different central masses were grown over the time interval 0 to 15 for MQ98 and 0 to 28 in my models. The dashed curves on the right shows results from reruns of two cases using the SCF method.

pioneering work by May, van Albada \& Norman (1985) and most recently by Merritt \& Quinlan (1998, hereafter MQ98).

While the simulations by MQ98 appear to have been performed with considerable care, one aspect of their results seemed highly surprising. As shown in Figure 1 (left), the shape of their initially triaxial model responded gradually to the introduction of a small central mass, and continued to evolve for many tens of dynamical times after the central mass reached a steady value at $t=5$ for $\mathrm{M}_{h}=0.3$ and $t=15$ for the two lower mass cases. I suspected that this behavior was a numerical artifact, possibly caused by their use of the so-called SCF method proposed by Hernquist \& Ostriker (1992): although the basis set of functions is complete, truncation to a comparatively few terms provides a severe restriction on the complexity of shapes which can be represented. Perhaps their numerical technique couples shape changes at different radii, which have greatly differing orbital time scales, rendering their simulations unable to sustain a shape which is axisymmetric near the center while remaining non-axisymmetric in the outer parts. I therefore decided to check their work by following their procedure as closely as possible but employed a different method to compute the gravitational field. The code, which closely resembles that previously described by McGlynn (1984) and requires somewhat less computer time than the SCF method (Sellwood 1997), expands the 
mass distribution in surface harmonics on a 1-D grid of shells, thus avoiding any coupling between the shape of the gravitational field at different radii.

My results (Figure 1, right) are markedly different from those reported by MQ98 (Figure 1, left). I find that the original triaxial shape both evolves rapidly as the central mass rises and stops evolving as soon as the increase stops at $t \sim 28$. The rate of evolution in the two lower central mass models is clearly slower in the simulations by MQ98, whereas I find even more rapid evolution (not shown) when I grow the central mass as fast as did MQ98.

Reverting to an independent implementation of the SCF method with the same number of terms gives the results shown by the dashed lines in Figure 1 (right). These lines track the solid curves as the central mass rises, but the later diverging trend, most obvious for the smallest central mass, shows that indeed the shape to continues to evolve after the central mass stops growing when the SCF code is used. This behavior confirms my suspicions that the SCF method to compute the gravitational field caused the slow evolution reported by MQ98. I have made a number of other tests, varying the usual numerical parameters of time step, softening length of the central mass, etc., which have made no difference to the behavior. While the slower initial evolution reported by MQ98 remains unexplained, the continuing shape changes in their models appear to result from a numerical artifact.

Black hole masses may be smaller than first believed (e.g. Merritt \& Ferrarese 2001) and their host galaxies therefore able to remain strongly triaxial. I find that masses $\lesssim 0.1 \%$ have a negligible effect on the global shape for as long as I run the calculations.

The effect of a central mass on rapidly-rotating, triaxial bar is perhaps even less well understood. The mass of a central black hole alone is unlikely to be large enough to affect the global shape, but augmented by a stellar cusp and other concentrations of stars and gas, the central masses can be enormous. There is general agreement that large central masses will destroy a bar; a mass of $5 \%$ of that of the disk is clearly plenty (Norman et al. 1996), but Friedli (1994) claims that as little as 1\% is sufficient, while Sellwood \& Moore (1999) were able to grow a bar in a disk with a central mass larger than this! The criterion seems to be more complicated than just a simple mass fraction and the central density required to destroy a bar is not yet known.

\section{The Origin of Bars}

It is now clear that we do not understand the origin of bars in galaxies. The original prejudice (e.g. Sellwood \& Wilkinson 1993) that they were probably created by the well-known global instability (Miller et al. 1970; Hohl 1971; 
Kalnajs 1972) now seems untenable for two reasons.

First, most bright galaxies should be stable because they have dense bulges. My own recent work (Sellwood 1985, 1999; Sellwood \& Evans 2001) has established beyond doubt the correctness of Toomre's (1981) idea that an entire disk can be stable if it has a dense center. A disk with a rotation curve that stays high close into the center, even if fully-self-gravitating, has no tendency to form a bar; early simulations simply lacked the dynamic range in density and time-scales to show this. However, a problem now arises for barred galaxies because they too have inner mass distributions which ensure a strong inner Lindblad resonance (ILR) that should have prevented the bar from forming. Indications of strong ILRs in nearby barred galaxies include nuclear rings (Buta \& Crocker 1993; Benedict et al. 1999), ring-like concentrations of molecular gas (e.g. Sakamoto et al. 1999; Jogee \& Kenney 2000) and offset dust lanes (Athanassoula 1992). Binney et al. (1991), Weiner \& Sellwood (1999) and others also find evidence for an ILR in the bar of the Milky Way, which seems inevitable given the central density distribution (Becklin \& Neugebauer 1968). This abundance of evidence is strongly suggestive, but indirect. Definitive verification requires detailed modeling of the mass distribution and velocity field within the galaxy, together with a determination of the pattern speed in each case (Lindblad, Lindblad \& Athanassoula 1996; Regan, Vogel \& Teuben 1997; Weiner et al. 2001) - all three cases reveal clear ILRs. The question raised by these observations is that if the bars have strong ILRs, which we now know should have inhibited the bar instability, how did the bars form in the first place? It is possible the central density rose after the bar formed, but the mass inflow required to produce such strong ILRs may well have destroyed the bar.

Second, bars seem to have formed long after their host disks came into existence. There appears to be deficiency of bars in galaxies at $z>0.5$ (van den Bergh et al. 1996; Abraham et al. 1999), suggesting that bars form late in the evolution of a disk galaxy. While some bars could be missed because of bandshifting (e.g. Bunker et al. 2000), a marked deficiency remains (van den Bergh et al. 2000, 2001). Thus most bars appear to form long after their host disks were assembled, which requires a slow, secular process quite different from the rapid dynamical instability.

I have observed an alternative mode of bar formation in number of simulations which remains consistent with both these properties and might conceivably be the way bars form in nature. It is well known that spiral patterns remove angular momentum from stars near the inner Lindblad resonance. The damping of the wave in second order perturbation theory (Kalnajs 1971; Lynden-Bell \& Kalnajs 1972; Mark 1974) depends on the assumption that the 
perturbation is too weak to trap the stars, but larger amplitude patterns can cause stars to be trapped. Simulations (Sellwood 1981; Sellwood \& Moore 1999) show that trapping in this way leads to bar which grows in length and strength in an episodic manner with each favorable pattern. It should be noted that this process differs from that proposed by Lynden-Bell (1979) because it relies on rapid changes in the angular momenta (or actions) of stars.

Is this the way real bars are formed? Further work is in hand to test whether the mechanism can account for the observed fraction of bars, the distribution of bar strengths and whether the resulting bars have properties consistent with those observed.

\section{Dynamical friction on bars}

Even though we do not understand their origin, bars allow us to impose powerful constraints on the dark matter (DM) content of the inner regions of galaxies. Weiner et al. (2001) show how the non-axisymmetric flow pattern driven by a bar can be used to determine the M/L of the bar and disk; they find, for the case of NGC 4123, that the stars must contribute essentially all the central attraction to account for the orbital speeds in the inner parts of that galaxy, and that the central density of DM must be low. Their powerful argument, however, does not belong in this review because it involves no $N$-body simulations.

Debattista \& Sellwood $(1998,2000)$ use $N$-body simulations to obtain a similar constraint on the inner DM content of barred galaxies. Tremaine \& Weinberg (1984a) argued that a bar rotating in a dense DM halo would lose angular momentum to the halo through dynamical friction, and Weinberg (1985), using perturbation theory, showed that the time scale for bar slow-down could be short (a few bar rotations).

There is strong, but not overwhelming, evidence to indicate that bars in real galaxies are fast - i.e. corotation lies only just beyond the end of the bar. Merrifield \& Kuijken (1995), Gerssen et al. (1999) and Debattista \& Williams (2001) apply the method of Tremaine \& Weinberg (1984b) to three barred galaxies, finding in all cases that the bar is fast. Modeling the gas flow yields the pattern speed less directly; our work on NGC 4123 (above), and that of Lindblad et al. (1996) and Regan et al. (1997), again indicates that bars in real galaxies rotate rapidly. The position of dust lanes in bars suggests that fast rotation is generic (van Albada \& Sanders 1982; Prendergast 1983; Athanassoula 1992). Thus it appears that bars in real galaxies have not suffered much friction.

Our simulations confirmed Weinberg's prediction of rapid bar slow down 


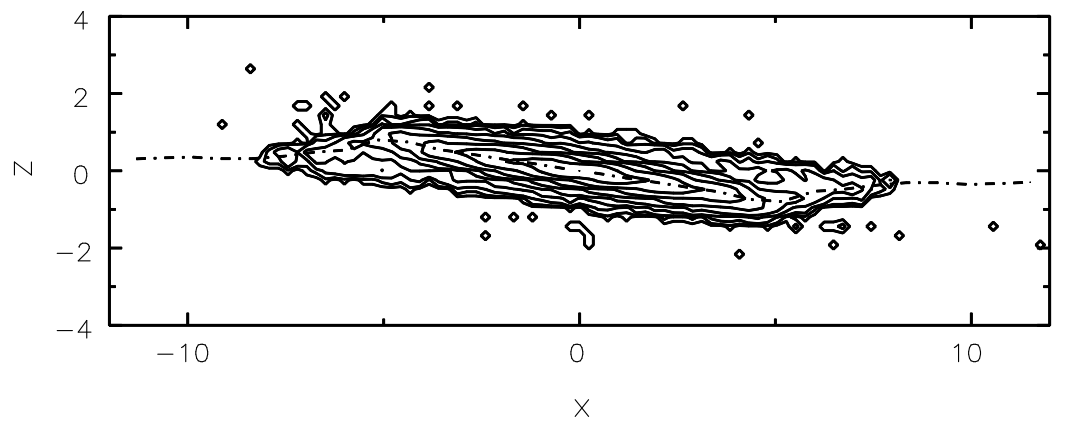

Figure 2: Contours of projected disk density after about 3 orbits at $R=5$ disk scales. By this time, the inner disk has tilted $\sim 10^{\circ}$ away from its original plane, which was horizontal. The dot-dash line indicates the cross-section of a layer of test particles, which should trace the expected locus of the HI layer.

when the halo has a moderate central density, unless the halo has large angular momentum in the same sense as the disk. Friction decreases the bar pattern speed, driving the corotation point out to unacceptable distances. The halo angular momentum required to avoid strong braking is unrealistically large, even when the halo is flattened to $b / a \simeq 0.5$ and rotation is confined to the inner halo only. Bars are therefore able to maintain their observed high pattern speeds only if the halo has a central density low enough for the disk to provide most of the central attraction in the inner galaxy.

\section{$5 \quad$ Friction-induced warps}

Debattista \& Sellwood (1999) found a quite different use for dynamical friction between the disk and halo: If the spin axes of the disk and of the DM halo are misaligned, dynamical friction will cause the angular momentum vectors to move closer towards alignment. This occurs even when the halo is not significantly flattened by rotation.

Their simulations of this process showed that the inner disk tips more rapidly than the outer disk, because its higher density induces a stronger frictional response. The outer disk therefore lags as the inner disk tips, creating a warp as shown in Figure 2. The inner tilt is straight while the warp has a leading twist, just as observed (e.g. Briggs 1990).

Note that the weak coupling between the inner and outer disk is an advantage in this mechanism, since it creates the warp, whereas it is the principal obstacle for would-be warp mode theories which founder because coupling is 
too weak to persuade the edge to precess at uniform rate (e.g. Toomre 1983).

The warp shown in Figure 2 does not last indefinitely; it is a transient response when the two spin vectors find themselves out of alignment. Fortunately, a duration of several galaxy rotations is plenty long enough to be consistent with the observed prevalence of warps, as it is likely the spin vector of the halo changes from time to time as a result of late minor mergers (e.g. Quinn \& Binney 1992), the natural consequence of any hierarchical theory of structure formation. (See also the contributions by Moore and by Bullock to these proceedings.)

It should be noted that random motions of stars in the plane provide a significant additional source of stiffness for a disk, one which is ignored in the usual treatment of the disk as a collection of wire rings coupled only by gravity. Random motion is important because adjacent mass elements of the disk share the same stars, and therefore cannot tip relative to each other as much as if gravity were the only restoring force. Debattista \& Sellwood (1999) present the only calculations so far to include this effect, and show that the stiffness of the disk rises markedly with the level of random motion of the disk stars.

\section{Acknowledgments}

I would like to thank V. Debattista for comments on a draft version. This work was supported by NASA LTSA grant NAG 5-6037 and by NSF grant AST-0098282.

\section{References}

Abraham, R. G., Merrifield, M. R., Ellis, R. S., Tanvir, N. \& Brinchman, J. 1999, MNRAS, 308, 596

Athanassoula, E. 1992, MNRAS, 259, 345

Becklin, E. E. \& Neugebauer, G. 1968, Ap. J., 151, 145

Benedict, G. F., Howell, A., Jorgensen, I., Chapell, D., Kenney, J. \& Smith, B. J. 1998, STSCI Press Release C98

Binney, J. J., Gerhard, O. E., Stark, A. A., Bally, J. \& Uchida, K. I. 1991, MNRAS, 252, 210

Briggs, F. H. 1990, Ap. J., 352. 15

Bunker, A., et al. 2000, astro-ph/0004348

Buta, R. \& Crocker, D. A. 1993, Astron. J., 105, 1344

Debattista, V. P. \& Sellwood, J. A. 1998, Ap. J., 493, L5

Debattista, V. P. \& Sellwood, J. A. 1999, Ap. J., 513, L107

Debattista, V. P. \& Sellwood, J. A. 2000, Ap. J., 544, 704

Debattista, V. P. \& Williams, T. B. 2001, in Galaxy Disks and Disk Galaxies, eds. J. G. Funes SJ \& E. M. Corsini, ASP Conference Series 230, (San Francisco: Astronomical Society of the Pacific) p 553

Friedli, D. 1994, in Mass-Transfer Induced Activity in Galaxies, ed. I. Shlosman (Cambridge: Cambridge University Press) p 268 
Gerhard, O. E. \& Binney, J. 1985, MNRAS, 216, 467

Gerssen, J., Kuijken, K. \& Merrifield, M. R. 1999, MNRAS, 306, 926

Hernquist, L. \& Ostriker, J. P. 1992, Ap. J., 386, 375

Hohl, F. 1971, Ap. J., 168, 343

Jogee, S. \& Kenney, J. 2000, in Dynamics of Galaxies From the Early Universe to the Present, eds. F. Combes, G. A. Mamon \& V. Charmandaris, ASP Conference Series 197, (San Francisco: Astronomical Society of the Pacific) p 193

Kalnajs, A. J. 1971, Ap. J., 166, 275

Kalnajs, A. J. 1972, Ap. J., 175, 63

Lindblad, P. A. B., Lindblad, P. O. \& Athanassoula, E. 1996, A. \& A., 313, 65

Lynden-Bell, D. 1979, MNRAS, 187, 101

Lynden-Bell, D. \& Kalnajs, A. J. 1972, MNRAS, 157, 1

Mark, J. W-K. 1974, Ap. J., 193, 539

May, A., van Albada, T. S. \& Norman, C. A. 1985, MNRAS, 214, 131

McGlynn, T. A. 1984, Ap. J., 281, 13

Merrifield, M. R. \& Kuijken, K. 1995, MNRAS, 274, 933

Merritt, D. \& Quinlan, G. 1998, Ap.J., 498, 625 (MQ98)

Merritt, D. \& Ferrarase L. 2001, astro-ph/0107134

Miller, R. H., Prendergast, K. H. \& Quirk, W. J. 1970, Ap. J., 161, 903

Norman, C. A., Sellwood, J. A. \& Hasan, H. 1996, Ap. J., 462, 114

Prendergast, K. H. 1983, in Internal Kinematics and Dynamics of Galaxies, IAU Symp. 100, ed E. Athanassoula (Dordrecht: Reidel) p 215

Quinn, T. \& Binney, J. 1992, MNRAS, 255, 729

Regan, M. W., Vogel, S. N. \& Teuben, P. J. 1997, Ap. J., 482, L143

Sakamoto, K., Okamura, S. K., Ishizuki, S. \& Scoville, N. Z. 1999, Ap. J., 525, 691

Schwarzschild, M. 1979, Ap. J., 232, 236

Sellwood, J. A. 1981, A. \& A., 99, 362

Sellwood, J. A. 1985, MNRAS, 217, 127

Sellwood, J. A. 1997, in Computational Astrophysics, eds. D. A. Clarke \& M. J. West (San Francisco: ASP Conference Series 123), 215

Sellwood, J. A. 1999, in Galaxy Dynamics - A Rutgers Symposium, eds. D. Merritt, J. A. Sellwood \& M. Valluri (San Francisco: ASP) 182, p 351

Sellwood, J. A. \& Evans, N. W. 2001, Ap. J., 546, 176

Sellwood, J. A. \& Moore, E. M. 1999, Ap. J., 510, 125

Sellwood, J. A. \& Wilkinson, A. 1993, Rep. Prog. Phys., 56, 173

Toomre, A. 1981, in The Structure and Evolution of Normal Galaxies, ed. S. M. Fall \& D. Lynden-Bell (Cambridge: Cambridge University Press), p. 111

Toomre, A. 1983, in Internal Kinematics and Dynamics of Galaxies, IAU Symposium 100, ed E. Athanassoula (Dordrecht: Reidel) p 177

Tremaine, S. \& Weinberg, M. D. 1984a, MNRAS, 209, 729

Tremaine, S. \& Weinberg, M. D. 1984b, Ap. J., 282, L5-L7

van Albada, T. S. \& Sanders, R. H. 1982, MNRAS, 201, 303

van den Bergh, S. et al. 1996, Astron. J., 112, 359

van den Bergh, S. et al. 2000, Astron. J., 120, 2190

van den Bergh, S., Cohen, J. G. \& Crabbe, C. 2001, astro-ph/0104458

Weinberg, M. D. 1985, MNRAS, 213, 451

Weiner, B. J. \& Sellwood, J. A. 1999, Ap. J., 524, 112

Weiner, B. J., Sellwood, J. A. \& Williams, T. B. 2001, Ap. J., 546, 931 